\title{
Tecnologias Interativas e Processos de Criação: Experiências de Aprendizagem Transdisciplinares Associadas a um Hackerspace
}

\author{
Interactive Technologies and Creative Processes: Transdisciplinary Learning Experiences Associated \\ with a Hackerspace
}

\author{
Erica Azevedo da Costa e Mattos \\ Universidade Federal de Santa Catarina, Brasil \\ ericamattos@gmail.com \\ Diego Fagundes da Silva \\ Universidade Federal de Santa Catarina, Brasil \\ diegofagundes@gmail.com
}

José Ripper Kós

Universidade Federal de Santa Catarina, Brasil

josekos@gmail.com

\begin{abstract}
The article presents an experience of association between a hackerspace and a multidisciplinary design studio at the Department of Architecture and Urbanism of the Federal University of Santa Catarina, during two semesters. The Tarrafa Hacker Club members shared their collaborative environment with the students and tutors throughout the academic experiment. The hacker culture highlighted dynamic relations that connect people, information, tools and matter. This approach has suggested meaningful speculations about the future of architecture education and practice.
\end{abstract}

Keywords: Hackerspace; Multidisciplinarity; Creative process; Collaborative environment; Interactive technology.

\section{Introdução}

O desenvolvimento tecnológico vem associado a aceleradas modificações não apenas nos aspectos mais globais de organização social, econômica e política mundial, como também nos aspectos mais íntimos do indivíduo, tais como os modos de perceber, pensar e agir sobre o mundo. Consequentemente, tais transformações repercutem também nas nossas relações de produção, criação e aprendizagem que são hoje colocadas sob novos prismas. A cultura hacker emerge nesse contexto como uma manifestação de variadas posturas críticas a respeito da nossa realidade tecnologicamente mediada. Uma das manifestações mais recentes desta cultura tem-se dado através da disseminação do fenômeno dos hackerspaces. Em um primeiro momento podemos definir hackerspaces como lugares físicos operados comunitariamente, na figura de laboratórios ou oficinas, onde pessoas podem se reunir e trabalhar em seus projetos, frequentemente vinculados à tecnologia.

Nesse artigo apresentaremos a disciplina "Ateliê Livre Tecnologias Interativas e Processos de Criação", uma experiência piloto desenvolvida no curso de Arquitetura e Urbanismo da Universidade Federal de Santa Catarina, associada ao processo de consolidação do hackerspace Tarrafa Hacker Clube, que desempenhou um importante papel no desenvolvimento da disciplina compartilhando a infraestrutura necessária. Infraestrutura é aqui entendida não como um conjunto estático composto pelo espaço físico, ferramentas e equipamentos, mas como um processo ativo, através das relações dinâmicas que são estabelecidas entre pessoas, informações, ferramentas e envolvimento material (Eriksson, 2011).

Ademais, ressaltamos que a cultura hacker específica que nos remete o movimento dos hackerspaces - e o nosso recorte empírico do Tarrafa Hacker Clube - não deve ser vista como representativa da totalidade de posturas e práticas associadas a esse termo. Apesar do termo consolidar um campo abrangente que compartilha determinados comprometimentos éticos, técnicos e também ideológicos, a diversidade de práticas verifica diferenças até mesmo divergentes entre si. Desta forma, trataremos das características que se relacionam ao movimento dos hackerspaces, cientes da sua parcialidade e especificidades.

\section{Cultura Hacker e Hackerspaces}

Apesar da cultura hacker como conhecemos hoje e dos termos hacker e hacking terem suas origens nos laboratórios de computação entre as décadas de 1950 e 1960 no MIT (Massachusetts Institute of Technology), encontramos seus princípios também em práticas dissociadas deste contexto estritamente computacional.

Linus Torvalds, um dos principais desenvolvedores por trás do sistema operacional Linux, aponta que "computer hackers themselves have always admitted this wider applicability of their 
ways. [...] In this sense, a person can be a hacker without having anything to do with computers." (Himanen, 2002, p. ix). Ou, nas palavras de Otto von Busch, artista e pesquisador em design: "hacking is an expanding a field of action, for many." (Busch \& Palmås, 2006, p. 28).

Para Busch (2009) hacking - ou o "hackeamento", em uma possível tradução para o português - pode ser definido como uma prática de intervenção direta, na qual identifica três características principais. Primeiramente, o hackeamento trata de uma habilidade de abrir um sistema, acessá-lo e assim aprender a dominar seus circuitos, defesas e estruturas. Ele é também uma tática específica para reivindicar e alterar esse sistema, acoplandose a ele e redirecionando seus fluxos para um objetivo mais desejável, construindo um novo sistema melhorado. Por último, os métodos, técnicas e ferramentas presentes no hackeamento são compartilhados livremente entre os envolvidos, de modo que qualquer interessado possa mudar ou desenvolver novas aplicações.

O conceito de hackeamento torna-se particularmente explícito e relevante para nós ao analisarmos práticas que caracterizam um hackerspace. Em seu estudo sobre hackerspaces, Eriksson (2011) categoriza as atividades encontradas por ele em três grupos, que, de certa forma, abarcam a conceituação de hackeamento trabalhada por Busch e ainda ampliam o campo de ação. O primeiro grupo por ele identificado como "modificação de sistemas fechados" trata do tradicional significado de hackeamento, em concordância com a definição de Busch e, basicamente se refere à compreensão, modificação e ampliação de funcionalidades de um dado sistema. Já o segundo grupo "composição através de meios simples", diz respeito ao processo de criação fazendo o uso de componentes e elementos básicos (ex: sensores e atuadores) frequentemente obtidos através de sucata e outros objetos. Como terceiro grupo de atividades, a "experimentação com hardwares e softwares de código aberto" reflete o uso crescente de dispositivos de código aberto como o Arduino e os kits de robôs e impressoras 3d para a elaboração de novos projetos.

Schrock (2011) aponta que os indivíduos que frequentam os hackerspaces não podem ser uniformemente classificados, são bastante heterogêneos em suas motivações para o uso do espaço. Tais indivíduos possuem ainda diferentes formações e experiências que podem variar dos campos tradicionalmente relacionados à tecnologia até às artes e ciências humanas. Ainda segundo o autor, uma identidade coletiva define as especificidades de cada hackerspace e é gerada pelos interesses momentâneos de seus membros, suas atividades e eventos em comum.

Para Eriksson (2011), os hackerspaces são autogerados e não determinados por uma lógica externa - surgem quando pessoas com diferentes objetivos e motivações se unem através de uma prática comum e do compartilhamento de recursos e conhecimento. Eriksson (2011) coloca também que as composições sociais relacionadas aos hackerspaces e aos recursos que são fornecidos por eles na forma de tecnologia e oportunidades locais definem condições para uma forma de criação radicalmente diferente da maneira industrial de desenvolvimento tecnológico, da maneira formal para aprender a tecnologia e da maneira padrão de viver com a tecnologia. Para ele, ao invés de serem vistos como um meio para cumprir objetivos claros previamente definidos, hackerspaces devem ser vistos como lugares onde metas, motivações e desejos podem ser explorados, descobertos e construídos.

Os primeiros hackerspaces sugiram na Alemanha na década de 90 . Em poucos anos se espalharam pela Europa, quando em 2007, o modelo dos hackerspaces europeus foi importado para os Estados Unidos e ganhou grande força e popularidade com a fundação do NYC Resistor (Nova York) e do Noise Bridge (São Francisco). Hoje o hackerspace movement possui um alcance global, com muitos espaços independentes operados comunitariamente em diversos países. No Brasil o primeiro hackerspace, o Garoa Hacker Clube, surgiu em 2010 em São Paulo e abriu caminho para a criação de dezenas de hackerspaces no Brasil, incluindo o Tarrafa Hacker Clube em Florianópolis.

\section{O Tarrafa Hacker Clube}

O Tarrafa Hacker Clube é um hackerspace em formação cuja estrutura serviu de base para o desenvolvimento da experiência pedagógica piloto relatada por este trabalho. Foi iniciado em 2012 e carrega grande influência do Garoa HC, explícita em seu estatuto de fundação. O Tarrafa HC, assim como outros hackerspaces, iniciou suas atividades em uma lista de discussão virtual ainda sem contar com um espaço físico. As primeiras atividades presenciais se deram na forma de palestras organizadas no intuito de agregar pessoas interessadas e difundir o conceito de hackerspace. Em dado momento, já com um pequeno grupo regular formado, encontros temáticos começaram a acontecer na forma de oficinas em espaços variados como coworkings e casas dos envolvidos. Posteriormente surgiu a possibilidade de compartilhar uma sala no Departamento de Arquitetura e Urbanismo da UFSC, vinculandose à disciplina recém criada "Ateliê Livre - Tecnologias Interativas e Processos de Criação". O funcionamento do Tarrafa HC é em muitos aspectos semelhante ao funcionamento de outros hackerspaces, conta com atividades regulares que acontecem por meio de encontros, projetos livres e também com atividades específicas entre as quais podemos situar as palestras e oficinas.

Em outras palavras, trata-se de um espaço gerado e mantido por seus membros (atualmente uma associação sem fins lucrativos) onde a prática crítica e criativa acontecesobretudo em torno da tecnologia e da curiosidade. O acesso ao Tarrafa HC é garantido por seu caráter aberto ao público em geral e seus membros compartilham espaço, ferramentas e conhecimentos, o que retoma o conceito de 


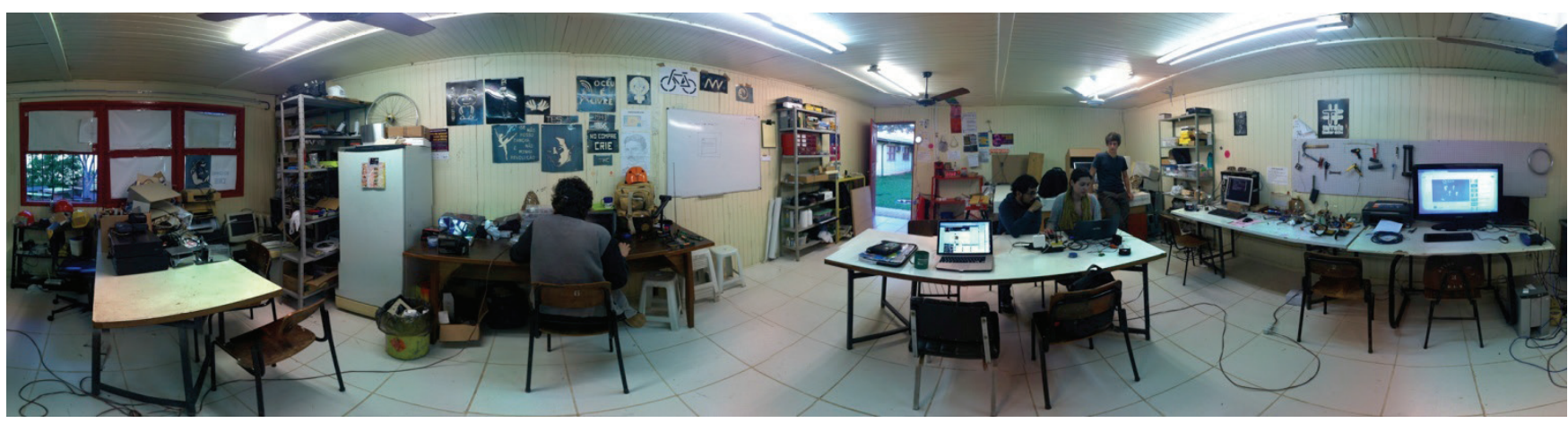

Figura 1: Imagem panorâmica do espaço físico ocupado pelo Tarrafa HC, 2013. Foto: Daniel Spillere Andrade.

infraestrutura dinâmica e relacional. Tal infraestrutura ao mesmo tempo em que serviu ao desenvolvimento da disciplina relatada a seguir, serviu-se também dela a ponto de poder, com isso, consolidar sua presença física.

\section{Ateliê Livre - Tecnologias Interativas e Processos de Criação}

A disciplina "Ateliê Livre - Tecnologias Interativas e Processos de Criação" foi criada com o intuito de fazer um cruzamento entre as práticas desenvolvidas em um hackerspace, neste caso o Tarrafa $\mathrm{HC}$, e o ensino de projeto em arquitetura, urbanismo e design. A presente experiência foi proposta como uma nova disciplina optativa dentro da grade do curso de Arquitetura e Urbanismo da Universidade Federal de Santa Catarina (UFSC). Dentro do escopo do presente artigo não iremos discutir o resultado dos trabalhos realizados pelos estudantes, e sim os processos que geraram tais projetos e como a relação com o Tarrafa HC influenciou o seus desenvolvimentos.

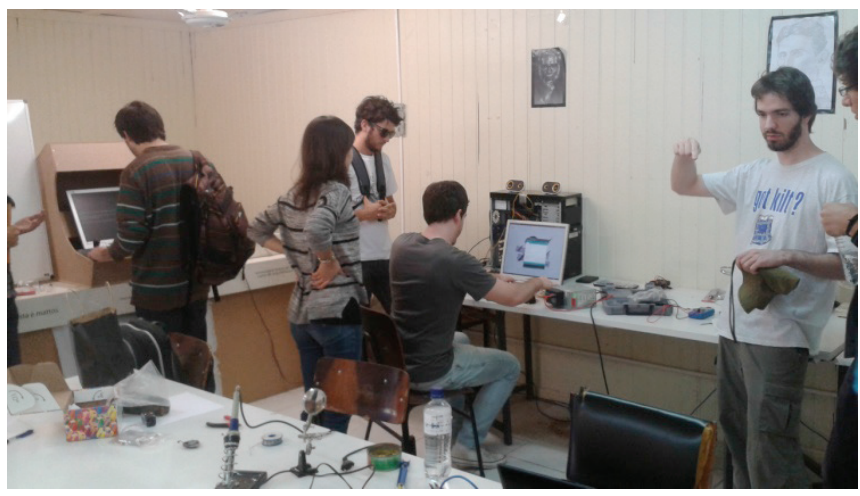

Figura 2: Estudantes da turma 2013/1 durante aula no espaço do Tarrafa HC, 2013. Foto: autores.

A experiência, realizada durante dois semestres letivos, tomou como base outras práticas realizadas em contextos institucionais diversos. Podemos citar como principal referência a disciplina "Atelier Integrado de Arquitetura" ministrada na Universidade Federal de Minas Gerais (UFMG), com o suporte do laboratório LAGEAR, coordenado pelo professor José dos Santos Cabral Filho.
Entretanto, diferentemente da disciplina oferecida pela UFMG, a experiência retratada no presente artigo não ficou restrita aos alunos do próprio curso (a disciplina da UFMG é parte do currículo obrigatório do curso de Arquitetura e Urbanismo e oferecida aos alunos ingressantes), mas estimulou também a participação de estudantes de outras áreas como Ciências da Computação, Engenharias Elétrica, Eletrônica e de Automação. O caráter transdisciplinar foi dado pela conjugação de saberes complementares vindos de diferentes áreas na construção de uma experiência compartilhada que resultou ao fim de cada semestre na produção e documentação de instalações interativas em espaços públicos dentro da universidade.

A escolha do tema em torno das instalações se deu essencialmente como um mote para a realização de intervenções físicas e espaciais utilizando recursos disponíveis dentro da estrutura oferecida pelo Tarrafa HC. Neste sentido, mais do que a discussão sobre princípios e teorias a respeito da inserção de uma instalação como uma obra de arte em um espaço público foi dada a prioridade ao discurso do fazer, pensar e produzir em grupo e em um ambiente colaborativo. Neste sentido as instalações foram tomadas como dispositivos de estímulo à criatividade para a solução de um problema comum. O objetivo de cada grupo, todavia, foi totalmente definido por seus membros, assim como a escolha dos recursos, tecnologias e conhecimentos a serem utilizados.

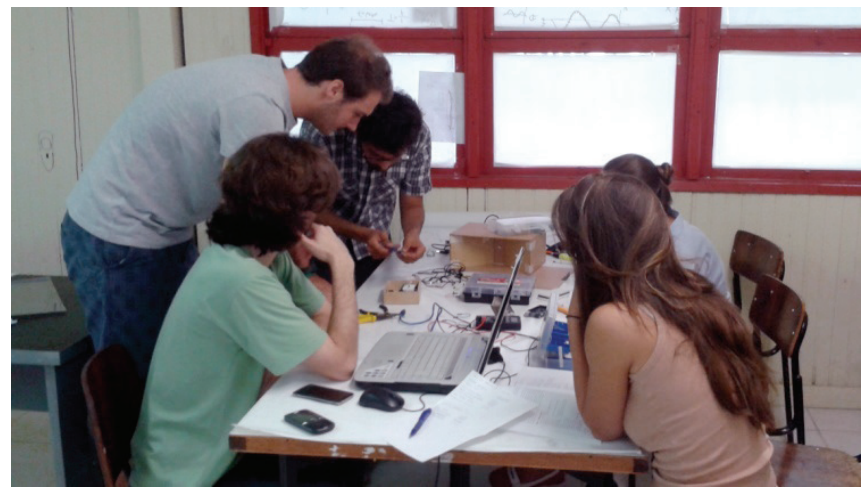

Figura 3: Estudantes da turma 2012/2 durante a aula no espaço do Tarrafa HC, 2012. Foto: autores. 
Nos dois semestres em que a disciplina foi oferecida os métodos de trabalho foram semelhantes, com a realização inicial da apresentação das intenções pedagógicas e de aulas e oficinas práticas para uma instrumentalização básica para o uso das tecnologias sugeridas - Arduino, componentes eletrônicos simples e sucata - que foram logo seguidas pelo desenvolvimento do trabalho em grupo final. Paralelo a esse desenvolvimento foram mantidos dois espaços virtuais, um blog da disciplina e um grupo de discussões no Facebook.

No primeiro semestre, com um número menor de alunos matriculados, optou-se por trabalhar em um único grupo no qual a atuação dos professores, autores do presente trabalho, se deu de forma mais intensa, ajudando no processo de trabalho e na tomada de decisões necessárias.

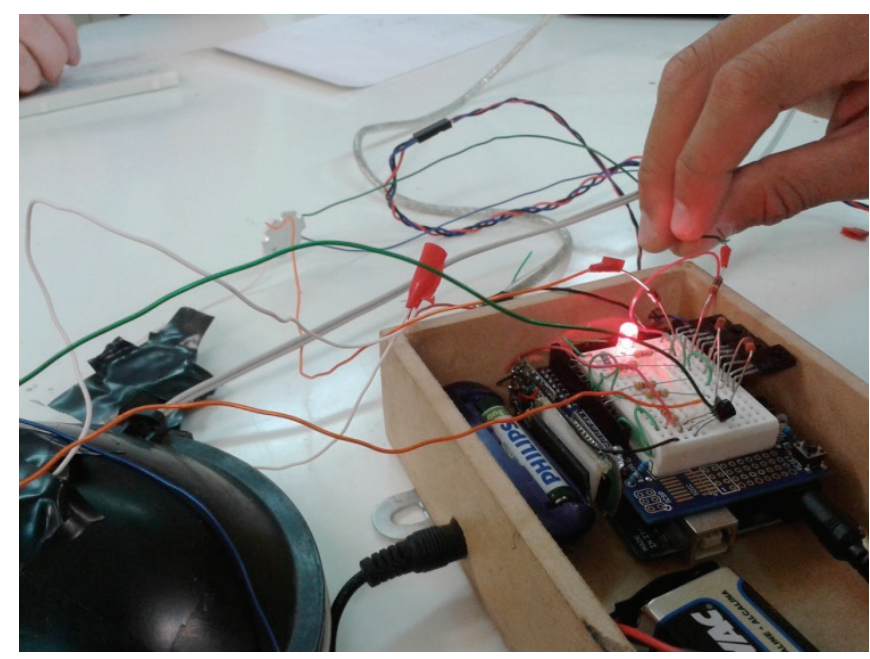

Figura 4: Protótipo do trabalho em grupo da turma 2012/2 com Arduino e componentes reutilizados. Foto: autores.

No segundo semestre, com mais alunos, originados de grande variedade de departamentos, foram formados 3 grupos que desenvolveram trabalhos independentes. As propostas desenvolvidas nessa segunda edição da disciplina foram direcionadas a um espaço mais próximo e acessível, as dependências do próprio curso de Arquitetura, de forma a facilitar o desenvolvimento de maior número de trabalhos. Neste semestre, as decisões sobre como desenvolver os projetos em grupo couberam mais aos alunos, sendo a participação dos professores mais como gestores do espaço.

O desenvolvimento da disciplina no Tarrafa HC favoreceu os estudantes a se tornarem protagonistas ativos da produção e compartilhamento do conhecimento e a figura do professor transformou-se do papel de mestre explicador para facilitador do processo de aprendizagem dentro dessa estrutura colaborativa não hierárquica. (Rancière, 2002)

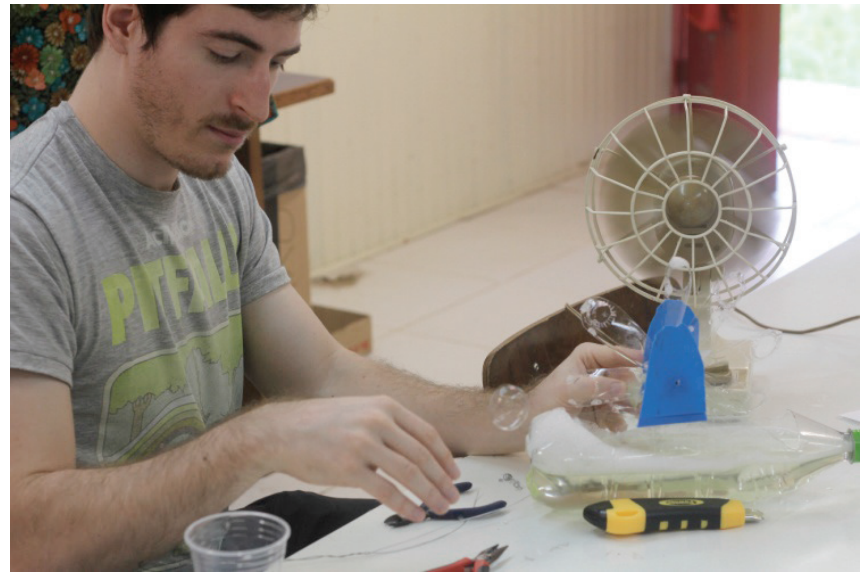

Figura 5: Desenvolvimento de um dos trabalhos da turma 2013/1, 2013. Foto: autores.

\section{Considerações}

Percebemos que as características identificadas nos hackerspaces são facilmente encontradas nas atividades do Tarrafa HC que se mantém independente da universidade, porém não foram totalmente transpostas para a experiência da disciplina devido as limitações associadas ao caráter acadêmico inerente a esta. A participação livre e espontânea dos membros do Tarrafa HC e dos demais interessados em suas atividades ao mesmo tempo gera e é usufruída pela a infraestrutura dinâmica e relacional do espaço. Por mais que tenhamos identificado um interesse genuíno nos estudantes da disciplina, o calendário de entregas e os encontros em grupo necessários que iam além do horário limitado de aula se demonstraram como empecilho para o desenvolvimento dos trabalhos e da exploração dos meios e ferramentas.

Por outro lado, percebemos também que a infraestrutura do Tarafa HC refletiu fortemente no processo de trabalho dos estudantes e foi uma das facetas da disciplina mais valorizadas pelos mesmos segundo relatos coletados ao final de cada semestre. Concluímos que esta infraestrutura teve um impacto positivo, mostrando e promovendo outra abordagem possível aos processos de projeto. Identificando os modelos abstratos dos procedimentos e processos presentes nos hackerspaces, podemos incorporá-los às práticas profissionais e de aprendizagem em arquitetura, design e urbanismo.

Podemos tirar lições em dois níveis distintos: como uma forma de apropriação alternativa das tecnologias disponíveis e do desenvolvimento tecnológico, e também como uma apropriação mais ampla dos valores encontrados - assumindo ações mais abertas, descentralizadas, compartilhadas e colaborativas, através de intervenções diretas motivadas pelos interesses e curiosidades, mesmo que não orientadas a uma finalidade específica.

Por fim, gostaríamos de apontar a importância de iniciativas multidisciplinares envolvendo experimentos relacionados à tecnologia, estimulando a formação de uma base de comunicação entre estudantes e profissionais de diferentes áreas do conhecimento. 


\section{Agradecimentos}

À CAPES (Ministério da Educação) e ao CNPq (Ministério da Ciência, Tecnologia e Inovação) pelo apoio aos pesquisadores. Aos membros do Tarrafa Hacker Clube, por oferecer o apoio necessário à realização da experiência, especialmente aos colaboradores Daniel Spillere Andrade e Ramiro Polla e aos estudantes participantes.

\section{Referências}

Busch, O. von, \& Palmås, K. (2006). Abstract hacktivism : the making of a hacker culture. London; Istanbul: Open Mute.
Busch, O. von. (2009). Engaged Design and the Practice of Fashion Hacking: The Examples of Giana Gonzalez and Dale Sko. Fashion Practice, 1(2), 163-186.

Eriksson, M. (2011). Labbet utan egenskaper. Retrieved from http://blay.se/papers/labbet.pdf

Himanen, P. (2002). The Hacker Ethic: A Radical Approach to the Philosophy of Business (Reprint.). Random House Trade Paperbacks.

Rancière, J. (2002). O Mestre Ignorante: Cinco Lições Sobre a Emancipação Intelectual. Belo Horizonte: Autêntica.

Schrock, A. R. (2011). A Hackerspace primer: Hackers, Makers, and Teachers (Part 2 - identities). Andrew Richard Schrock. Retrieved from http://andrewrschrock.wordpress.com/2011/08/03/ahackerspace-primer-hackers-makers-and-teachers-part-2-identities/ 